\title{
Resúmenes del V Encuentro Nacional de Medicina del Dormir
}

\section{Relación del síndrome apnea hipopnea obstructiva del sueño con obesidad e impacto de la titulación con CPAP/ BPAP evaluados con videopolisomnografía}

Pella-Cruzado VM, * Alanis-Guevara I, $\neq$ Vázquez $F^{\ddagger}$

*Hospital Ángeles del Pedregal, México, D.F. ${ }^{\ddagger}$ Centro Médico Nacional de Occidente, Guadalajara, Jalisco, México.

Introducción: El síndrome de apnea hipoapnea obstructiva del sueño (SAHOS) es un trastorno crónico, progresivo, frecuentemente asociado a enfermedades metabólicas, cardiovasculares y cerebrovasculares, con elevado riesgo laboral y accidentes de tránsito, mala calidad de vida, sueño y una mortalidad elevada. Afecta al 5\% de la población adulta y al $2 \%$ de niños. Ocasionada por una disminución del calibre de la vía aérea respiratoria superior. El diagnóstico se basa en la sospecha clínica y en la realización de polisomnografía completa. El tratamiento consiste en el uso de presión continua positiva del aire (CPAP). Pacientes y métodos: Estudio descriptivo, retrospectivo, transversal de 100 pacientes enviados por sospecha de apnea del sueño, roncopatía e hipersomnolencia, a la Clínica del Sueño del Hospital Ángeles del Pedregal (HAP) Ciudad de México, para efectuarles estu- dio de video-polisomnográfico (VPSG) nocturno, entre junio 2010-junio 2013, población adulta, enviados por médicos otorrinolaringólogos, neumólogos, cardiólogos, internistas y neurólogos. Resultados: El 82\% de pacientes eran masculinos, edad promedio 53 años con un rango de 21 a 86 años, mayor de 65 años $21 \%$. El motivo de envío fue por ronquido en $37 \%$, apnea en $39 \%$. El tiempo promedio del padecimiento fue de 9.5 años con un predominio mayor a 30 años en el $83 \%$ de casos. La comorbilidad se encontraba asociada a hipertensión arterial en el 57\%, síndrome metabólico 64\%, enfermedad cardíaca $16 \%$ y diabetes en $13 \%$, sobrepeso y obesidad $90 \%$, con un índice de masa corporal (IMC), escala de Mallampati grado III y IV en el $75 \%$. La VPSG demostró apnea obstructiva en el $70 \%$, central $31 \%$ y mixta en el $26 \%$, grado severo de apnea en $82 \%$ y moderado en $15 \%$. El promedio de la fase 3-4 de sueño fue del $12 \%$ y para el REM fue del $10 \%$, valores que se duplicaron de inmediato con la aplicación del CPAP nasal a una presión media de $8 \mathrm{cmH}_{2} \mathrm{O}$ con una disminución significativa de la apnea severa al $20 \%$. Conclusiones: Existe un retraso en el diagnóstico y tratamiento de los pacientes con SAHOS con una media de 10 años. La obesidad estuvo presente en el 90\%. El tratamiento con CPAP mejora las fases de sueño, sueño profundo y sueño REM con un claro beneficio hemodinámico y cardiovascular por registros comparativos pre y postratamiento.

\section{Apnea central del} sueño emergente al tratamiento: Utilidad de la titulación CPAP/BPAP evaluados con video polisomnográficos
Pella-Cruzado VM, *Alanis-Guevara I, Vázquez $F^{\ddagger}$

\begin{abstract}
*Hospital Ángeles del Pedregal, México, D.F ${ }^{\ddagger}$ Centro Médico Nacional de Occidente, Guadalajara, Jalisco, México.
\end{abstract}

Introducción: El síndrome de apnea central del sueño emergente al tratamiento (SACET) de las apneas obstructivas, se caracteriza por la aparición de apneas centrales y/o respiración de Cheyne Stokes, posterior a la titulación con presión continua de aire (CPAP). Actualmente se propone la utilización de la servo ventilación (ASV). El presente trabajo pretende demostrar la diferencia de respuesta demostrada con VPSG, la utilidad del CPAP vs. BPAP como la mejor alternativa de tratamiento. Material y metódos: Estudio descriptivo, retrospectivo, transversal de 195 pacientes con SAHOS, del 2012 al 2014, 14 pts. titulados con CPAP y 14 con BPAP, de la Clínica del Sueño del Hospital Ángeles del Pedregal, Ciudad de México. Resultados: El 89\% (n25) fueron hombres, la edad media fue 58 años, $32 \%$ (n9) mayores de 65 años. Se utilizó protocolo AAMS (JCSM 2008), noche dividida, la presión promedio del CPAP $8 \mathrm{cmH}_{2} \mathrm{O}$, BPAP media IPAP 9 EPAP $6 \mathrm{cmH}_{2} \mathrm{O}$. La prevalencia SACET fue de $14.3 \%$. Comorbilidad: hipertensión arterial en $73 \%$ (n19), enfermedad cardíaca 30\%, DM2 en $11 \%$, IMC sobrepeso u obesidad $82 \%$ (n23); escala Mallampati con grado III-IV en $75 \%$ (n21). Sin tratamiento (ST): eficacia del sueño $70 \%$, índice de apnea hipopnea (IAH) 69/Hr, grado severo $86 \%$ (n24). Síndrome de apnea hipopnea obstructiva del sueño (SAHOS) 50\%, síndrome de apnea central $45 \%$, apnea mixta $53.5 \%$ (15), arousals 94\%, PLMS 100\% (28). Con VPSG titulación: CPAP eficacia del sueño $88 \%$, IAH 26xhxs. Severa y moderada $36 \%$, apnea central (SAC) $100 \%$ (14), obstructiva (SAOS) 64\% (9), Cheyne Stokes (RCS) 57\% (8). BPAP: Eficacia del sueño $75 \%$, IAH $17 x$ xxs, severa $14 \%$, moderada $29 \%$, SAHOS $28 \%$ (8), SAC $28 \%$ (8), RCS 21\% (6). Conclusión: La apnea obstructiva severa desaparece con 
ambos tratamientos, persistiendo en grado moderado en un quinto de nuestra población con un mejor resultado a expensas de CPAP $14 \%$ vs. BPAP 21\%. La apnea severa en general presenta una mejoría notable con CPAP $(36 \%)$ comparado con BPAP (50\%).

\section{Manejo de la apnea del sueño sin alta probabilidad pretest o con comorbilidades mediante tres noches de poligrafía respiratoria domiciliaria}

\author{
Guerrero-Chapa AA, * Embid $C$, \\ Isetta $V, \S$ Farre $R, \S$ Durán-Cantolla J," \\ Parra O" \\ *Hospital Universitario «Dr. José Eleuterio \\ González». UANL. Monterrey, México; Hospital \\ Clinic. UB-IDIBAPS. Barcelona, España. \\ ¥Hospital Clinic. UB-IDIBAPS. Barcelona, \\ España; CIBER de Enfermedades Respiratorias \\ (CIBERES). Madrid, España. \\ ${ }^{\circledR}$ CIBER de Enfermedades Respiratorias \\ (CIBERES). Madrid, España; Unidad de \\ Biofísica y Bioingeniería. Facultad de \\ Medicina. IDIBAPS; Universidad de \\ Barcelona. Barcelona, España. \\ "CIBER de Enfermedades Respiratorias \\ (CIBERES). Madrid, España; Instituto de \\ Investigación y Unidad de Investigación \\ Clínica Bio-Araba; Hospital Universitario \\ Araba. Vitoria, España. \\ "CIBER de Enfermedades Respiratorias \\ (CIBERES). Madrid, España; Hospital Sagrat \\ Cor. Barcelona, España.
}

Introducción: El diagnóstico de la apnea obstructiva del sueño (OSA) utilizando métodos simplificados como la poligrafía respiratoria domiciliaria, sólo se recomienda en pacientes con alta probabilidad pretest. Objetivo: Determinar la eficacia diagnóstica, decisión terapéutica y costos del diagnóstico de OSA utilizando polisomografía (PSG) $v s$. tres estudios consecutivos de poligrafía respiratoria en pacientes con sospecha clínica leve-moderada o con comorbilidades que puedan enmascarar los síntomas de OSA. Material y métodos: Estudio aleatorizado, ciego, cruzado, de tres noches de PR (3N-PRD) vs. PSG. La eficacia diagnóstica fue evaluada con curvas ROC. Las decisiones terapéuticas para evaluar la concordancia entre las dos diferentes estrategias fueron realizadas por médicos especialistas en sueño y neumólogos (staff y residentes) utilizando «agreement level» y coeficiente kappa. Se consideraron los costos de cada estrategia diagnóstica. Resultados: Se seleccionaron 56 pacientes. El Epworth fue de 10.1 (5.3) puntos. El gráfico Bland Altman para IAH mostró buena concordancia. Las curvas ROC mostraron la mejor AUC en pacientes con IAH PSG $>5,[0.955(\mathrm{CI}=0.862 \mathrm{a}$ 0.993)]. Para un IAH PSG $\geq 5$, un IAH PRD de 5 puede efectivamente excluir y confirmar el diagnóstico de OSA. Para un IAH PSG $\geq 15$, un IAH PRD $\geq 22$ puede confirmar y un IAH PRD $<7$ puede excluir OSA. Las mejores decisiones terapéuticas fueron obtenidas por los especialistas en medicina del sueño (81.8\%). La mejor costo-eficacia diagnóstica fue obtenida con 3N-PRD. Conclusiones: Tres noches consecutivas de poligrafía respiratoria domiciliaria evaluadas por un especialista en medicina del sueño pueden ser usadas para el manejo de pacientes sin alta probabilidad clínica pretest de OSA o con comorbilidades.

\section{Efecto de humidificador sobre eventos adversos y adherencia a CPAP en pacientes con SAHS}

\section{Baños-Flores MR, Carrillo-Alduenda JL, Castillo A, Castorena-Maldonado A, Anaya-Ramírez SN}

INER Ismael Cosío Villegas, Ciudad de México

Antecedentes: El dispositivo de presión positiva continua en la vía aérea (CPAP) es el tratamiento de elección para el síndrome de apnea hipopnea del sueño (SAHS); utilizarlo con un humidificador es una estrategia que podría incrementar la adherencia; sin embargo, su efecto no es claro e incrementa costos. El objetivo es comparar los eventos adversos reportados por el paciente y el apego a CPAP con y sin humidificación entre pacientes con SAHS. Materiales y métodos: Se analizó la base de datos de la Clínica de CPAP del Instituto Nacional de Enfermedades Respi- ratorias Ismael Cosío Villegas, Ciudad de México, se incluyeron pacientes con SAHS, habitantes de esta ciudad, con al menos 3 meses de tratamiento con CPAP; excluimos pacientes con traqueotomía y otros trastornos respiratorios del sueño; se aplicó un cuestionario estandarizado de síntomas y efectos adversos. Resultados: Se incluyeron 277 pacientes, 184 con humidificador y 93 sin humidificador, sin diferencias en variables antropométricas, presión terapéutica y marcadores de gravedad. No se encontró diferencia en las horas/uso/ promedio/día entre los pacientes con y sin humidificador $(6.3 \pm 0.4 v s .6 .8 \pm 0.12, \mathrm{P}$ $=0.3$ ). No hubo diferencias entre los grupos al reportar algún evento adverso, sequedad de nariz/boca y cefalea matutina; aquellos que usaron humidificador reportaron más fuga (19\% vs. 36\%, respectivamente, p $=0.006)$. El reporte de mejoría subjetiva entre los grupos no tuvo diferencias $(85 \%$ vs. $90 \%, \mathrm{p}=0.93$ ), pero el grupo con humidificador suspendió en menor frecuencia su tratamiento después de 3 meses (19\% vs. $0.05 \%, \mathrm{p}=0.001)$. El uso de humidificador no incrementó la probabilidad de reportar mejoría (OR 1.04, 0.33-3.22 IC95\%), pero sí disminuyó la probabilidad de abandonar el tratamiento a 3 meses (OR 0.26, 0.11-0.61 IC95\%). Conclusiones: En pacientes con SAHS en tratamiento con un dispositivo CPAP el humidificador no disminuye los eventos adversos e incrementa el reporte subjetivo de fuga, pero reduce la probabilidad de abandono a 3 meses.

\section{Síntomas relacionados al dormir en pacientes con cáncer de pulmón localmente avanzado (etapas IIIA y IIIB)}

\section{García-Sancho FMC, ${ }^{*}$ Reyes-Zuñiga \\ $M,{ }^{*}$ Torre-Bouscoulet $L,{ }^{*}$ Arrieta $O,{ }^{\neq}$ Martínez-Briseño D, ${ }^{\star}$ Fernández-Plata $R$, * Pérez-Padilla $J R^{*}$}

*INER Ismael Cosío Villegas, Ciudad de México; ‡Instituto Nacional de Cancerología.

Introducción: Los pacientes con etapas avanzadas de cáncer pulmonar sufren de problemas relacionados al dormir que afectan negativamente la calidad del 
sueño. (Akyuz RG, 2013). Hipótesis: Los pacientes con cáncer pulmonar tendrán una elevada prevalencia de síntomas relacionados al dormir. Objetivo: Investigar la prevalencia de síntomas asociados al dormir, malestar psicológico, dolor, disnea y calidad de vida en los pacientes con cáncer pulmonar localmente avanzado. Material y métodos: Se incluyeron consecutivamente a todos los pacientes con diagnóstico de cáncer pulmonar localmente avanzado del Instituto Nacional de Cancerología que tuvieron indicación para radioterapia y que consintieron en participar en el proyecto. Los síntomas relacionados al dormir se obtuvieron del Índice de Calidad de Sueño de Pittsburg. Además, se aplicaron la escala de disnea MRC (Medical Research Council) y calidad de vida (EUROQL). Número de aprobación del Comité de Investigación del INER C12-12. Resultados: Se incluyeron 35 pacientes cuya mediana de edad fue de 61 (28-85) años. $45.7 \%$ fueron hombres y $57.1 \%$ fueron fumadores. El $91.4 \%$ de los pacientes refirieron síntomas relacionados al sueño siendo los más frecuentes la somnolencia diurna (60\%), el despertar nocturno (51.4\%), la presencia de dolor (45.7\%) y el tener una duración de sueño corta ( $<7$ horas) $42.9 \%$. El $71.4 \%$ de los pacientes refirieron tener alguna enfermedad asociada. Las más frecuentes fueron la gastritis $(41.2 \%)$ y la hipertensión arterial (31.4\%). El 63\% refirió cualquier síntoma de mala calidad de vida. Las más frecuentes fueron la presencia de dolor $(48.6 \%)$, la dificultad para realizar las actividades cotidianas $(28.6 \%)$ y la presencia de ansiedad o depresión (22.9\%). Conclusiones: Los pacientes con cáncer pulmonar refieren una elevada frecuencia de síntomas asociados al dormir, principalmente el dolor y una duración del sueño corta. Las alteraciones del sueño pueden estar relacionadas más a los síntomas del cáncer. El mejorar la disnea podría mejorar la calidad del sueño.

\section{Características de la población enviada a la Clínica de Sueño del Hospital General de México}

Díaz-Riveros MA, Mares-Gutiérrez $Y$, Bautista-Herrera D, Septien-Stute $L A$

Hospital General de México "Dr. Eduardo Liceaga"

El síndrome de apnea obstructiva del sueño (SAOS) es un problema de salud pública por su alta prevalencia y morbimortalidad. Es necesario conocer las características de nuestra población para establecer estrategias de prevención, diagnóstico y tratamiento. El objetivo del estudio es describir las características clínicas de los pacientes referidos a la Clínica de Sueño del Hospital General de México. Estudio observacional, descriptivo, transversal. Se incluyeron a todos los pacientes referidos a la clínica en el período 2010-2013. Se incluyeron a 303 pacientes, el $67.4 \%$ del género femenino. Se observó una elevada prevalencia de obesidad mórbida (60.4\%). Se documentó somnolencia excesiva en $28.6 \%$. Se identificó hipoxemia por oximetría de pulso en $22.2 \%$ e hipercapnia en $28.6 \%(n=63)$. El $77.3 \%$ contaba con estudio de sueño. La mayoría de los pacientes presentó AOS grave $(56.6 \%)$. El apego al tratamiento es bajo, tanto en el aspecto nutricional como al oxígeno medicinal $(58 \%$ y $28 \%$, respectivamente). El $24.8 \%$ de los pacientes con AOS cuenta con CPAP, pero con apego adecuado (93.2\%). El tiempo promedio desde la sospecha hasta el diagnóstico de AOS fue de 99.3 días y la mediana hasta la compra del CPAP de 269 días (37-1321). Se sometieron a cirugía bariátrica 65 pacientes, $83 \%$ con bypass gástrico y el $17 \%$ a manga gástrica. La mediana de pérdida de peso fue de $30 \mathrm{~kg}$. La prevalencia de la obesidad mórbida y las alteraciones en el intercambio gaseoso es alta en nuestra población, mientras que el apego al tratamiento es bajo probablemente a consecuencia de los altos costos del oxígeno medicinal y los dispositivos para el tratamiento de la enfermedad. Es necesario implementar nuevas estrategias para la prevención de la obesidad e identificar precozmente a los pacientes en riesgo de la enfermedad.

\section{Calidad de sueño en niños asmáticos}

García-Colín E, Vázquez-García JC, Carrillo-Alduenda JL, Del Razo-Rodríguez R, Ruiz-Martínez E

INER Ismael Cosío Villegas, Ciudad de México

Introducción: Los niños asmáticos tienen exacerbaciones nocturnas, por lo que la calidad de sueño puede estar relacionada con la gravedad del mismo. Pregunta de investigación: ¿Cómo es la calidad de sueño en niños con asma estable? Objetivo principal: -Describir la calidad de sueño en niños con asma estable. Objetivos secundarios: -Determinar la prevalencia de los principales trastornos del dormir. Investigar la asociación entre la gravedad del asma y la presencia de ronquidos y apnea del sueño. Material y métodos: Se incluyeron a todos los pacientes de 2 a 12 años con diagnóstico médico de asma, como casos prevalentes atendidos en el Servicio de Neumología Pediátrica del INER. Estables al menos cuatro semanas previas a su inclusión. Se diseñó en dos etapas: 1. Encuesta. Llenándose cuestionario de salud general, síntomas de asma y hábitos de sueño, así como antecedentes generales como obesidad, hipertrofia amigdalina y enfermedad por reflujo gastroesofágico). Espirometría en mayores de 6 años y medidas antropométricas. 2. Estudios de sueño. Se seleccionaron pacientes con ronquido habitual que completaron 2 semanas de seguimiento. Llenaron un diario de síntomas y flujometría, se realizó polisomnografía (PSG) y oximetría de pulso nocturna (SnoreSat). Para valorar las diferencias entre los distintos grupos de gravedad del asma se aplicó la prueba de Kruskal-Wallis para muestras no paramétricas, aquellos valores de $\mathrm{p}<$ 0.05 fueron considerados con significancia estadística. Resultados: Se reclutaron un total de 222 niños que cumplieron con los criterios de inclusión y exclusión. Edad promedio de 7 años, 133 del sexo masculino (60\%) y 89 del sexo femenino (40\%). Un 34\% fueron preescolares y el $66 \%$ escolares. Se encontró que $20.2 \%$ de los niños son roncadores habituales y se estimó una prevalencia de apnea de $6.2 \%$. Se reclutaron 45 niños $(20.2 \%)$ considerados por sus padres como roncadores habituales y se les realizó polisomnografía 
estándar nocturna, 16 fueron clasificados con asma intermitente (AI), 12 con asma leve persistente (ALP), 12 con AMP y 6 con ASP. No se observan diferencias en la eficiencia de sueño, cambios de etapa de sueño y latencia a sueño MOR, como tampoco hay diferencia en la arquitectura del sueño entre los distintos grupos de severidad del asma. Conclusiones: La gravedad del asma afecta la calidad de sueño referida por los padres. Los niños con asma grave aumentan el tiempo de vigila después de haberse dormido, y tienen una latencia anormalmente corta a sueño. La saturación de oxígeno durante el dormir no se modifica con la gravedad del asma.Los niños asmáticos tienen una elevada prevalencia de ronquido habitual y apnea del sueño.

\section{Presión positiva} autoajustable preoperatoria en niños con apnea obstructiva del sueño

\section{Arango-Gopar E, Reyes-Zúñiga M, Torre-Bouscoulet L, Castorena- Maldonado A, López-Escárcega E \\ INER Ismael Cosío Villegas, Ciudad de México}

Introducción: La presión positiva autoajustable en la vía aérea (APAP) ha sido poco explorada como tratamiento preoperatorio en niños con apnea obstructiva del sueño (AOS) grave. El objetivo es caracterizar a una población de niños que fueron tratados con APAP mientras esperaban la fecha quirúrgica (adenoamigdalectomía) y describir la frecuencia de complicaciones. Métodos: Estudio retrospectivo. Datos de pacientes entre 2 y 13 años, con AOS grave, en tratamiento con APAP (ResMed, Autoset S7, Sydney, Australia), sometidos a adenoamigdalectomía. El diagnóstico de AOS se estableció mediante poligrafía respiratoria (Remmers Sleep Recorder, Sagatech, Calgary, Alberta, Canadá). Se registraron complicaciones quirúrgicas. Se utilizó estadística descriptiva. Resultados: Datos de 54 pacientes, 34 hombres, edad 5.8 años (2-13). Hubo 23 niños $(42.5 \%)$ con obesidad. Duración de sueño $10 \pm 1.2$ h•noche-1. Síntomas: 44 (81.4\%) presentaron ronquido habitual, $45(83.3 \%)$ apneas observadas, 26 (48.1) somnolencia, $35(64.8 \%)$ resistencia para dormir, 34 $(62.9 \%)$ fueron vigilados mientras dormían y 28 (51\%) tuvieron irritabilidad. El índice de eventos respiratorios (IER) fue $37.7 \pm 26.3$. Se identificó a 15 pacientes (28\%) con asma, $6(11 \%)$ con reflujo gastroesofágico y 7 (13\%) con trastorno por déficit de atención. El perfil de uso de APAP mostró que el promedio de horas de uso por noche fue $5.12 \pm 2.9$, IER residual $6.7 \pm 3.5$ y percentila 95 de presión 10.1 $\pm 2.9 \mathrm{cmH}_{2} \mathrm{O}$. Fueron 36 niños $(66.6 \%)$ que utilizaron APAP $>4$ h $\bullet$ noche-1. Dos pacientes $(3.7 \%)$ tuvieron complicaciones (hipertensión arterial y sangrado). No identificamos factores relacionados a las horas de uso de APAP ni asociaciones entre complicaciones y uso de APAP. Conclusiones: La mayoría de los niños con AOS grave son hombres, obesos y con otros estados comorbidos (asma el más frecuente). Aunque se requieren ensayos clínicos controlados, la baja frecuencia de complicaciones sugiere que el tratamiento preoperatorio con APAP es una estrategia clínicamente útil en niños con AOS grave.

\section{Adherencia a CPAP en pacientes con síndrome de apnea obstructiva del sueño de acuerdo al método diagnóstico. Poligrafía respiratoria vs. Polisomnografía}

\section{López-Escárcega E, Reyes-Zúñiga $M$, Castorena-Maldonado A, García-Polo L, Torre-Bouscoulet L}

INER Ismael Cosío Villegas, Ciudad de México

Introducción: Existe información limitada acerca de la adherencia a CPAP de pacientes con síndrome de apnea obstructiva del sueño (SAOS), dependiendo del método diagnóstico; poligrafía respiratoria (PR) vs. polisomnografía (PSG). Los objetivos fueron comparar si la adherencia a CPAP difiriere cuando el diagnóstico se establece por PR vs. PSG. Además, exploramos los factores relacionados a adherencia. Métodos: Estudio retrospectivo de la base de datos institucional de la clínica de CPAP del período comprendido de septiembre 2001 a julio 2012. Fueron adultos, portadores de SAOS, con prescripción de CPAP. La decisión del método diagnóstico (grupo PR vs. grupo PSG) fue establecida por el médico tratante. Para el análisis estadístico se utilizó STATA 12. Resultados: Datos de 1,852 pacientes (68\% hombres), edad $50 \pm 13$ años, índice de masa corporal (IMC) $35.9 \pm 7.8 \mathrm{~K} \cdot \mathrm{m}-2$, Epworth $13.5 \pm 7$, con seguridad social 546 (29.5\%). Grupo PR $=690$ pacientes (37\%); grupo PSG = 1,162 (63\%). El índice de eventos respiratorios fue $55.8 \pm 29.7$ y $66 \pm 39.5$ eventos.hr-1 en los grupos PR y PSG, respectivamente ( $<<0.0001)$. El IMC fue $33.9 \pm 5.7 \mathrm{~K} \cdot \mathrm{m}-2$ en el grupo PR vs. $37.2 \pm 8.5$ en el grupo PSG $(\mathrm{p}<$ 0.0001). El tratamiento con CPAP fue suspendido con mayor frecuencia en el grupo PSG vs. PRS (17.3\% vs. $12.7 \%, \mathrm{p}=$ 0.04). No hubo diferencias en la frecuencia de inicio de CPAP, presión prescrita ni en el tiempo transcurrido para iniciar el tratamiento. La mediana de inicio de tratamiento en todo el grupo fue de 28 días. Con relación a la adherencia (mediana de 476 días), la prueba de Log-Rank no mostró diferencias entre los grupos PR vs. PSG $(\mathrm{p}=0.63)$. La puntuación de Epworth fue el único factor relacionado a adherencia (OR 1.03; 1.01-1.05, p=0.02). Conclusión: En condiciones de la «vida real», la adherencia a CPAP es similar en pacientes diagnosticados por PR o PSG. La somnolencia fue el único predictor de adherencia.

\section{Hipoventilación y apnea central del sueño en un escolar con distrofia miotónica tipo 1}

Rodríguez-Tejada A, Torres-Fraga MG, Carrillo-Alduenda JL, Baños-Flores MR, Galicia-Polo ML, Reyes-Zúñiga MM

INER Ismael Cosío Villegas, Ciudad de México

Antecedentes: La distrofia miotónica tipo 1 (DM-1) es una enfermedad hereditaria relacionada al cromosoma 19. 
Se caracteriza por debilidad muscular y manifestaciones sistémicas asociadas, como alteraciones en el centro respiratorio y trastornos del sueño. Reporte de caso: Masculino de 8 años con diagnóstico de DM-1 referido por somnolencia diurna, cefalea matutina y ronquido. Aún deambula, acude a escuela regular y no se encontró insuficiencia respiratoria diurna, saturación de 94\% y ETCO2 de 36 mmHg al aire ambiente. Espirometría sugestiva de restricción, PIMAX de $20.09 \mathrm{cmH}_{2} \mathrm{O}$, PeMax de $31.5 \mathrm{cmH}_{2} \mathrm{O}$. La polisomnografía demuestra apnea central sin respiración de Cheyne Stokes, hipoxemia e hipoventilación durante el sueño por CO2 máximo de $53 \mathrm{mmHg}$ e índice de apnea central 39.3/h. Para estabilizar el patrón respiratorio, resolver la hipercapnia y la hipoxemia durante el sueño se ajustó binivel ST con presión soporte de 11 y frecuencia respiratoria de 16x'. Comentario y conclusiones: El mecanismo más conocido de apnea central es la inestabilidad respiratoria asociada a hiperventilación que alcanza el umbral apneico. En teoría, la hipoventilación limitada al sueño protegería de presentar apneas centrales. Este paciente presenta claras y poco comunes alteraciones del control central de la respiración a muy temprana. La identificación de trastornos durante el dormir permitirán el adecuado manejo y reducción de la morbimortalidad asociada.

\section{Adherencia y efectos secundarios al tratamiento con CPAP a presión alta en adultos con apnea del sueño e hipoventilación por obesidad}

\section{Castorena-Maldonado A, Orozco- González N, López-Escárcega E, Carrillo-Alduenda JL, Galicia-Polo L, Torres-Fraga $M$}

INER Ismael Cosío Villegas, Ciudad de México

Introducción: Hay poca información sobre la frecuencia y uso de CPAP a presiones mayores de $14 \mathrm{cmH}_{2} \mathrm{O}$ en pacientes con apnea del sueño (SAOS) e hipoventilación por obesidad (HO), el algoritmo de ajuste de presión positiva durante la polisomnografía de la Academia Americana de Medicina del Sueño recomienda cambiar de CPAP a un modo de ventilación Binivel si no se han conseguido los objetivos de la titulación a presión de $15 \mathrm{~cm}$ de $\mathrm{H}_{2} \mathrm{O}$; sin embargo, no hay sustento suficiente en la literatura. Objetivo: Conocer la frecuencia y adherencia al tratamiento con CPAP a presión alta y compararla con un grupo control de CPAP a presiones comunes. Material y métodos: Restrospectivo, análisis de la base de datos de la clínica de CPAP. Material y métodos: Se formaron dos grupos de comparación, aquellos con presión alta ( $\left.\geq 15 \mathrm{cmH}_{2} \mathrm{O}\right)$ y control $(<$ $15 \mathrm{cmH}_{2} \mathrm{O}$ ), la adherencia se midió de acuerdo con el número de horas por noche y el número de noches por semana. Resultados: Incluimos 2,387 pacientes adultos, el $34 \%$ fueron mujeres, el $85.4 \%$ con SAOS y $14.6 \%$ con HO. Se identificaron 451 casos (18.9\%) con presión alta. La adherencia del grupo presión alta fue de $7.14 \pm 1.95$ h con $6.7 \pm 0.95$ noches y del grupo control fue de $6.58 \pm 1.84 \mathrm{~h}$ con 6.5 \pm 1.18 noches, diferentes estadísticamente $\mathrm{p}=0.00$, el $82 \%$ y $72 \% \mathrm{p}=0.00$ tuvieron algún tipo de efecto secundario, respectivamente, principalmente por la inteface pero no fue una causa de abandono del tratamiento. El índice de masa corporal fue la variable que más se asoció con alta presión (OR 1.04 IC95\% 1.04-1.07) en los pacientes con SAOS y la saturación de oxígeno promedio durante el estudio de sueño (OR 0.95 IC95\% 0.93-0.98) en los pacientes con HO. Conclusiones: La adherencia al tratamiento con CPAP a presión alta fue mayor en comparación con el grupo con presión $<15 \mathrm{cmH}_{2} \mathrm{O}$ y los efectos secundarios fueron menores en el grupo con alta presión.

\section{Perfil de tratamiento con binivel en pacientes de la Clínica de Sueño del INER}

Espinosa-Méndez DA, Torres-Fraga MG, Carrillo-Alduenda JL, CastorenaMaldonado A, López-Escárcega E, Castillo-Gómez AD

INER Ismael Cosío Villegas, Ciudad de México
Introducción: El uso de presión positiva no invasiva a largo plazo es útil en diversas enfermedades respiratorias crónicas y tiene dos vertientes: la presión continua (CPAP) y aquélla que proporciona presión diferencial entre inspiración y espiración (binivel, ventilación mecánica no invasiva, (VMNI)). En general, la CPAP se reserva para trastornos obstructivos de la vía aérea superior y la VMNI para problemas complejos que casi siempre involucran hipoventilación crónica. En 2005, países de la unión Europea reportaron que existen diferencias importantes en la prescripción. En nuestro servicio, la VMNI se utiliza desde 1999 pero no se ha descrito el perfil de uso. Objetivo: Describir el perfil de uso de VMNI en pacientes de la Clínica de Sueño del INER desde la creación de la clínica de CPAP. Métodos: Estudio retrospectivo de 12 años en pacientes de la Clínica de Sueño del INER con prescripción y uso de VMNI, identificados en la base de datos de la Clínica de CPAP, excluyendo aquellos codificados erróneamente y/o con expediente incompleto. Se utilizó estadística descriptiva de acuerdo al comportamiento de las variables, con programa SPSS versión 20. Resultados: Se encontraron 206 registros: $80.6 \%$ (166) adultos, 59.7\% (123) hombres, 98\% (203) han conseguido equipo. Las causas más comunes de VMNI fueron: síndrome de hipoventilación obesidad (SHO) 47.2\% (92), síndrome de apnea obstructiva del sueño (SAOS) y enfermedades neuromusculares (ENM) con $22.1 \%$ (43) y $19.5 \%$ (38), respectivamente. El 93\% (84) se titularon mediante polisomnografía, con mediana de IAH de 27.5 (p25-75 de 7 y 77.5), mediana de saturación promedio durante el sueño de $86 \%$ (P25-75 de 79 y 90) y presión soporte promedio de 6 (5-8) $\mathrm{cmH}_{2} \mathrm{O}$. El tiempo promedio que demoran en conseguir un equipo es de 17 semanas, se observó apego óptimo. Se han reportado 14 fallecimientos principalmente de causa respiratoria. Conclusiones: La prescripción de la VMNI ha incrementado en los últimos años. Que el apego no sea un problema sugiere la gravedad de los pacientes que reciben VMNI. 


\section{Síndrome de} hipoventilación alveolar central congénito: Presentación de 3 casos

\section{Herrera-Flores EH, Carrillo-Alduenda $J$, Torres-Fraga MG, Reyes-Zúñiga MM, Figueredo-Ballesteros $D$, Castorena-Maldonado $A$}

INER Ismael Cosío Villegas, Ciudad de México

El síndrome de hipoventilación alveolar central congénita (SHACC) es una enfermedad rara secundaria a la mutación genética PHOX2B que ocasiona ausencia de la respuesta ventilatoria a la hipercapnia y que se manifiesta por hipoventilación alveolar que empeora durante el sueño. Se presentan 3 casos de SHACC diagnosticados y tratados en la Clínica de Sueño del Instituto Nacional de Enfermedades Respiratorias. Caso 1: Hombre de 12 años de edad enviado por hipercapnia y Cor pulmonale posterior a neumonía, tenía: SpO2 diurna 57\%, EtCO2 diurno de 47 $\mathrm{mmHg}$ y EtCO2 nocturno de $94 \mathrm{mmHg}$; se inició tratamiento con binivel $\mathrm{S}$, se mantuvo estable por 9 meses y falleció 6 meses después de suspender ventilación mecánica no invasiva por insuficiencia respiratoria aguda secundaria a neumonía. No se realizó análisis genético. Caso 2: Hombre de 1 años 3 meses, enviado por hipercapnia, respuesta exagerada a benzodiazepinas y dificultad al retiro de ventilación mecánica invasiva posterior a una neumonía; $\mathrm{SpO} 2$ diurna 91\%, EtCO2 diurno $44 \mathrm{mmHg}$, EtCO2 nocturno $66 \mathrm{mmHg}$; actualmente estable y tratado con un binivel ST. No se realizó análisis genético. Caso 3: Hombre de 2 años 8 meses, enviado por hipercapnia e hipertensión arterial pulmonar sin causa aparente, $\mathrm{SpO} 2$ diurna 84\%, EtCO2 diurno $64 \mathrm{mmHg}$, EtCO2 nocturno $160 \mathrm{mmHg}$, PHOX2B positivo (genotipo 20/25). Clínicamente estable y tratado con un binivel ST. Conclusiones: El SHACC es una enfermedad rara, pero tiene un gran impacto sobre la salud de los niños que la padecen, el fundamento de su tratamiento es la ventilación mecánica y es potencialmente fatal. Los 3 casos que se presentan corresponden a hombres en diferentes etapas de crecimiento y desarrollo (1 lactante, 1 pre-escolar y 1 escolar), que posiblemente representan diferentes genotipos de la mutación PHOX2B, recibieron tratamiento con VMNI con resultados satisfactorios excepto en un caso por abandono.

\section{Acuerdo en la arquitectura de sueño y eventos respiratorios entre un monitor tipo I y un monitor tipo 2}

Herrera-Flores EH, Carrillo-Alduenda JL, Torres-Fraga MG, Reyes-Zúñiga MM, Galicia-Polo ML, Castorena-Maldonado A

INER Ismael Cosío Villegas, Ciudad de México

Introducción: El síndrome de apnea obstructiva del sueño es un problema de salud pública que tiene un enorme subdiagnóstico; el estándar de referencia para el diagnóstico es la polisomnografía atendida (monitor tipo 1). En los últimos años se han desarrollado los monitores tipo 2 (polisomnografía no atendida en domicilio) que podría hacer el diagnóstico más eficiente. El objetivo de este estudio fue medir el acuerdo en la arquitectura de sueño y en los índices de eventos respiratorios entre un monitor tipo $1 \mathrm{y}$ un tipo 2. Materiales y métodos: A un grupo de sujetos con sospecha de apnea obstructiva del sueño y un grupo de sujetos control se les realizó un registro simultáneo de 1 noche con un monitor tipo 1 (Grass Telefactor) y un monitor tipo 2 (Alice PDX Philips Respironics) de acuerdo a estándares internacionales. Resultados: Se incluyeron 46 sujetos, 25 hombres (54\%), mediana edad 32.5 (IIC 30-43), IMC 25.7 (IIC 23.6-30), cuello 38 (IIC 35-40). El coeficiente de correlación interclase (CCI) para las fases del sueño fue de $0.81,0.74,0.54$ y 0.78 para $\mathrm{N} 1$, $\mathrm{N} 2$, N3 y R, respectivamente; el CCI para índice de apnea-hipopnea (IAH) fue 0.98. Conclusiones: El acuerdo general entre los monitores en la arquitectura de sueño es moderado/bueno, la mejor correlación se presentó en N1 y la peor en N3. El acuerdo en el IAH es muy bueno.
Validez diagnóstica del índice de severidad del insomnio

\section{Jiménez-Genchi A, Armijo-de Alzúa M, Sandoval-Rincón M}

Instituto Nacional de Psiquiatría Ramón de la Fuente

Introducción: El índice de severidad del insomnio (ISI) es probablemente el instrumento de informe subjetivo que más se emplea tanto en la práctica clínica como en la investigación para la evaluación del insomnio. Además de mostrar una elevada confiabilidad, también se ha documentado su utilidad en la identificación del insomnio. En virtud de lo anterior consideramos necesario conocer el comportamiento de este instrumento en población mexicana. Hipótesis: El ISI muestra valores satisfactorios en indicadores de validez diagnóstica. Objetivo: Evaluar la validez diagnóstica del ISI a través de la estimación de su sensibilidad, especificidad, valor predictivo positivo (VPP) y negativo (VPN). Método: Se seleccionaron sujetos, con edad $>16$ años, mujeres u hombres que otorgaron su consentimiento para participar. Todos se sometieron a una entrevista semiestructurada para determinar la presencia de insomnio con base en el criterio A del DSM 5 , que fue seguida por el llenado del ISI. Resultados: Se incluyeron 107 sujetos, 32 sin insomnio y 75 con insomnio, entre los cuales no hubo diferencias significativas por género (\% mujeres: 53.2 vs. 61.3, respectivamente; ) ni edad (42.5 DE $15.1 \mathrm{vs}$. 43.9 DE 15.8). Con una puntuación de 9 se obtuvo el mejor balance, con una sensibilidad de $88 \%$ y una especificidad de $87 \%$. Para una prevalencia de insomnio del 10\%, el VPP fue de $43 \%$ y el VPN $98 \%$. Discusión: Una puntuación de $\geq 9$ en el ISI mostró el mejor balance entre sensibilidad y especificidad. Este valor es intermedio entre el propuesto empíricamente en un inicio (8) y el que recientemente se estableció en muestras de la comunidad y clínicas (10 y 11). Sin embargo, el porcentaje de sujetos correctamente diagnosticados es bajo.

\section{Apnea obstructiva del sueño y su relación con el síndrome metabólico}


Díaz-Riveros MA, Mares-Gutiérrez Y, Bautista-Herrera D, Septien-Stute LA

Hospital General de México "Dr. Eduardo Liceaga"

La apnea obstructiva del sueño (AOS) es una enfermedad asociada a múltiples comorbilidades. El síndrome metabólico (SM) se encuentra relacionado con mayor riesgo cardiovascular. No se ha establecido una relación directa entre el SM y la AOS; sin embargo, se ha observado incremento en la prevalencia del SM en pacientes caucásicos con AOS (40-70\%), pero no se cuenta con datos en población mexicana. El objetivo del estudio es describir la prevalencia del SM y su relación con el síndrome de AOS. Estudio observacional, descriptivo, transversal. Se incluyeron a los pacientes referidos a nuestra unidad en el período 2010-2013. Se consideraron significativos los resultados con una $\mathrm{p}<$ 0.05. Se incluyeron a 303 pacientes, 99 $(32.7 \%)$ eran hombres. La edad promedio fue de $44.3 \pm 12.3$ años, el índice de masa corporal de $43.5 \pm 9 \mathrm{~kg} / \mathrm{m}^{2}$. Se describieron 22 comorbilidades. Cada paciente tuvo en promedio $3.3 \pm 2$ comorbilidades con un máximo de 10 y un mínimo de 1 . El 52\% de los pacientes referidos fueron diagnosticados con SM. Se realizaron estudios de sueño en 235 pacientes. El 56.5\% tenía AOS grave, $30.6 \%$ leve-moderado y $12.7 \%$ eran sanos. No se observó diferencia en la prevalencia de SM en los tres grupos ni mayor descontrol en los diferentes componentes del SM. La obesidad (100\%) y alteración en el metabolismo de la glucosa (71.6\%) fueron las comorbilidades más frecuentes. A diferencia de otros estudios en población con obesidad mórbida, no se observó incremento en la prevalencia del $\mathrm{SM}$ en pacientes con AOS vs. sin AOS. La prevalencia del SM en pacientes mexicanos con AOS es similar a la reportada en la literatura mundial y mayor a la población sin obesidad mórbida. Hacen falta más estudios en nuestra población para corroborar nuestros resultados.

\section{Somnolencia diurna} excesiva y su asociación con la apnea obstructiva del sueño
Díaz-Riveros MA, Mares-Gutiérrez Y, Bautista-Herrera D, Septien-Stute LA

Hospital General de México "Dr. Eduardo Liceaga"

La apnea obstructiva del sueño (AOS) es un problema de salud pública. Un síntoma característico es la somnolencia diurna excesiva (SDE) que sin ser específico ni sensible se ha relacionado con la intensidad de la AOS. A menudo se confunde la somnolencia como predictor de la AOS. El objetivo del estudio es evaluar y analizar la asociación entre SDE y la AOS. Estudio observacional, descriptivo, transversal. Se incluyeron a todos los pacientes referidos a nuestra unidad en el período 2010-2013. Se encontró asociación estadísticamente significativa entre la ESE y el índice de apnea/hipopnea $(r=0.30)$ y la saturación mínima durante el estudio de sueño $(r=0.34)$. La prevalencia de SDE fue de $28.6 \%$ (mujeres $17.5 \%$ y hombres $11.2 \%$ ). La mayoría de los pacientes con SDE tenían AOS grave. La sensibilidad, especificidad, VPP y VPN de la SDE para diagnóstico de AOS fue de 30.1\%, $93.1 \%, 96 \%$ y $16 \%$, respectivamente. La curva COR tuvo un área bajo la curva de 0.63 (IC95\% 0.53-0.72). La mayoría de los pacientes enviados a la clínica reportaron buena calidad de sueño. El $11.3 \%$ de los pacientes refirieron tener dificultad para controlar el sueño durante el día por lo menos 1-2 veces por semana y el $9.3 \%$ señaló que el sueño frecuentemente interfiere en su capacidad para realizar su trabajo. Treinta y tres $(36.7 \%)$ de los pacientes manejan, registrándose 3 accidentes automovilísticos a causa de somnolencia. La SDE es común en la AOS grave; sin embargo, su correlación con la gravedad de la enfermedad es débil. La somnolencia también se relaciona con la menor saturación durante el sueño. En nuestra población, la SDE tiene un alto valor predictivo positivo pero su sensibilidad y el VPN son muy bajos.

\section{Disminución de alteraciones respiratorias en pacientes con SAOS mediante el uso de}

\section{dispositivo de avance madibular (DAM) evaluado con polisomnografía nocturna dividida (PND)}

\section{García-Campos E, Haro-Valencia $R$, Labra-Herrera A, Galicia-Polo L}

Clínica de Trastornos del Sueño, Universidad Nacional Autónoma de México

El manejo del SAOS está lleno de controversias iniciando desde la pugna por saber cuál es la especialidad que debe estar a cargo, hasta la elección del tratamiento. Es por ello la importancia del adecuado manejo multidisciplinario que permita brindar alternativas terapéuticas eficaces, aceptables y menos costosas. El dispositivo de avance mandibular (DAM) es considerando como una de ellas gracias a su mecanismo de acción que permite mantener abierta la vía aérea durante el sueño. Sin embargo, el manejo inadecuado de esta alternativa y la falta de seguimiento, han conducido en muchos casos al rechazo y fracaso terapéutico. Por lo que proponemos en esta investigación el manejo multidisciplinario de esta alternativa mediante criterios de evaluación, así como su seguimiento conjunto mediante PND, procurando con ello alcanzar un alto beneficio terapéutico y económico. Obejtivo: Evaluar mediante polisomnografía nocturna la eficacia del DAM mediante PND, en 30 pacientes con SAOS manejados en forma multidisciplinaria dentro de la Clínica de Sueño, UNAM. Metodología: Selección de 30 casos diagnosticados con SAOS, únicamente mediante evaluación física e historia clínica de sueño que cubrieran los criterios médicos y odontológicos para la colocación de DAM. El seguimiento clínico se realizó cada 15 días y tres meses posteriores a su colocación, se evalúo la eficacia del DAM mediante PND. Análisis estadístico realizado mediante T de Student para comparar medias de valores respiratorios. Resultados: Los resultados obtenidos en la PND muestran cambios significativos con el uso de DAM (IC:95\%-p < .05): Reducción en el IAH (22.45 a 4.63 eventos/h), índice de ronquidos (159.95 a 32.46 eventos/h), aumento de $\mathrm{SaO} 2(89.98 \%$ a $91.39 \%)$, Escala Epworth 
(14.4 a 4.6) así como también cambios significativos en la evaluación subjetiva del paciente y de la pareja. Conclusiones: El uso del DAM demostró ser un tratamiento eficaz al ser empleado en forma multidisciplinaria y valorado mediante PND consiguiendo disminuir los costos tanto en una alternativa terapéutica eficaz como en el método de evaluación

\section{Trastornos del sueño: Su relación y responsabilidad en estomatología pediátrica}

\section{Ledesma-Muñoz HS}

Universidad Michoacana de San Nicolás de Hidalgo, Universidad Nacional Autónoma de México

Actualmente en estomatología pediátrica hay un gran interés en las alteraciones respiratorias durante el sueño, en niños, adolescentes y se incrementa en la edad adulta. La apnea del sueño y otros síntomas de trastornos respiratorios tienen un efecto especialmente importante sobre los niños debido a que ellos requieren más tiempo de sueño normal que los adultos. La prevalencia de ronquido en la población pediátrica es de $79 \%$ y la de la apnea obstructiva del sueño es de 0.7-2.9\%. Las manifestaciones clínicas habituales de la apnea obstructiva del sueño comprenden respiración bucal crónica, ronquidos, agitación durante el sueño, seguidos o no de despertar frecuente. También se debe sospechar la presencia de la apnea obstructiva del sueño en niños con sudoración profusa, enuresis, anorexia, trastornos del aprendizaje y de la conducta, fracaso escolar y somnolencia. El ronquido nocturno es el factor de riesgo más importante para identificarla actualmente en estomatología pediátrica. A pesar de ser una patología habitual en los niños, no todos los pediatras interrogan sobre ella en la práctica clínica ni representa un motivo de preocupación para los padres del paciente. El objetivo de tratamiento para la AOS es mantener la amplitud de la vía para el paso del aire. La profesión estomatológica ha comenzado a desempenar un papel primordial en el diagnóstico y tratamiento del síndrome, lo que deja ver una oportunidad importante de expansión de nuestro campo potencial de asistencia clínica. Con un tratamiento adecuado, la apnea obstructiva del sueño y sus complicaciones suelen desaparecer en la mayoría de los pacientes en edad pediátrica. Con un tratamiento adecuado, la apnea obstructiva del sueño y sus complicaciones suelen desaparecer en la mayoría de los pacientes en edad pediátrica.

\section{Impacto en la progresión del volumen faríngeo con presión positiva en pacientes con SAHOS}

Talayero-Petra JA, CastorenaMaldonado A, Martínez-Briseño D, Schobert-Capetillo I

INER Ismael Cosío Villegas, Ciudad de México

Introducción: El síndrome de apnea hipopnea obstructiva del sueño (SAHOS) es el trastorno respiratorio del sueño con mayor prevalencia (3.2\%) en México. La faringe de los pacientes con SAHOS es más estrecha y colapsable, entre otros factores por edema y tejido graso. El tratamiento de primera elección es la presión positiva (CPAP), lo cual tiene un impacto positivo en la salud. A su vez, disminuye el edema de la vía aérea superior (VAS) evitando el colapso y el traumatismo continuo de los tejidos dados por el mismo. No existe ningún estudio que de forma objetiva mediante faringometría acústica relacione las dimensiones faríngeas en pacientes con SAHOS y los cambios que puede tener después del manejo con CPAP. Objetivos: Comparar el volumen faríngeo por faringometría acústica, tanto basal como semanalmente durante cuatro semanas de tratamiento con CPAP. Analizar su relación con el cumplimiento al dispositivo. Material y métodos: Se realizó un estudio de tipo prospectivo, descriptivo y longitudinal. Se incluyeron de forma consecutiva pacientes con diagnóstico de SAHOS con indicación de tratamiento con CPAP. Se realizó una faringometría acústica con el equipo Acoustic Rhinometry and Pharyngometry System marca
ECCOVISION $^{\circledast}$, bajo los lineamientos establecidos en el consenso de faringometría acústica. Esta prueba se realizó antes de iniciar tratamiento, así como de forma semanal durante cuatro semanas de uso de presión positiva con equipo CPAP autoajustable o presión fija, registrándose: áreas de sección transversa, área más estrecha y volumen faríngeo. Para comparar el efecto de adherencia al CPAP se definió como buen cumplimiento el uso mínimo de 4 horas por noche, al menos 5 noches de la semana. Las variables se expresaron de acuerdo a su tipo y distribución. La comparación entre variables se realizó con la prueba de $\mathrm{T}$ pareada y ANOVA para mediciones repetidas. Se empleó el estadístico STATA 12. Resultados: Se incluyeron 50 pacientes, 37 hombres (74\%) y 13 mujeres (26\%). De acuerdo a los valores semanales obtenidos a partir de la faringometría se observó un incremento en la media $33.21 \mathrm{cc}$ a $35.5 \mathrm{cc}$ del volumen a la cuarta semana $p=0.00$. Se evaluaron las características de uso y cumplimiento al equipo CPAP por paciente de forma semanal. El análisis de mediciones repetidas mostró ser diferente. La mayoría de los pacientes mostró tener un buen cumplimiento al CPAP. Conclusiones: El CPAP incrementa progresivamente el volumen faríngeo. El uso de CPAP aumenta el volumen y área de sección transversa a partir de dos semanas de uso del equipo. No se observó asociación relevante entre el cumplimiento al CPAP con el aumento en el área de sección transversa de la faringe.

\section{Subpoblaciones celulares del sistema inmune en tejido adenoamigdalino en niños con SAOS}

Figueredo-Ballasteros $D$, Rivera-Rosales RM, Sada-Ovalle I, Otáñez-Díaz J, Carrillo-Alduenda JL, Castorena-Maldonado $A$

INER Ismael Cosío Villegas, Ciudad de México

Introducción: El síndrome SAOS en niños se estima en $1 \%$ en la Ciudad de México, siendo la hipertrofia del tejido linfoide 
la principal causa, se ha sugerido al SAOS como una enfermedad inflamatoria, tanto local como sistémica y se conocen marcadores inmunohistoquímicos que tipifican las subpoblaciones celulares del sistema inmune. Objetivo: Evaluar la frecuencia de linfocitos T, CD4+, CD8+, NKT, linfocitos $\mathrm{B}$, monocitos/macrófagos y células plasmáticas en adenoides y amígdalas de los niños con SAOS grave. Material y método: Se incluyeron niños de 3 a 7 años con diagnóstico de SAOS y ronquido primario por PSG estándar. Se excluyeron niños con obesidad o comorbilidades. Se realizó adenoamigdalectomía. Se realizaron tinciones de inmunohistoquímica para CD 3, CD 4, CD 8, CD 20, CD 57, CD 68, kappa y lambda. Se dividieron los pacientes en 2 grupos: pacientes con y sin apnea obstructiva del sueño. Se compararon los grupos para cada marcador y se analizaron con la prueba exacta de Fisher. Resultados: Se incluyeron 17 niños, de los cuales 11 eran mujeres (64.7\%) y 6 hombres $(35.3 \%)$. Se dividieron en dos grupos: El primero 8 pacientes con apnea grave $(47 \%)$ y 9 con ronquido primario (53\%). Se encontró que no hay diferencia significativa entre los grupos en los diferentes marcadores, CD3, CD8, CD57, CD68, kappa y lambda. El $100 \%$ de los pacientes en ambos grupos mostraron CD20+ y CD4-. Conclusiones: En este estudio no se encontró diferencia en las poblaciones linfocitarias, macrófagos y células plasmáticas entre los pacientes con apnea obstructiva del sueño y los pacientes con ronquido primario, sugiriendo ausencia o poca influencia del SAOS; sin embargo, se encontró la presencia de CD20 en todos los pacientes sugiriendo una importante actividad de inmunidad humoral mediada por linfocitos B. Se requieren más estudios con herramientas de evaluación complementarias de la inmunidad.

\section{Efecto de la acupuntura,} sobre el patrón de sueño de ratas estresadas por inmovilización

Santana-Miranda $\boldsymbol{R},{ }^{*}$ RojasZamorano JÁ,, Domínguez-Salazar E,, Rodríguez-Gálvez A,, VelázquezMoctezuma $\mathrm{J}^{\ddagger}$

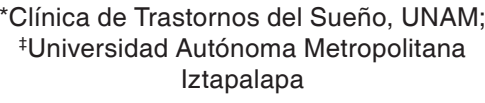

*Clínica de Trastornos del Sueño, UNAM; ‡Universidad Autónoma Metropolitana Iztapalapa

Antecedentes: Estresores determinados modifican la arquitectura del sueño, tal es el caso del estrés por inmovilización, el cual provoca un rebote de sueño MOR en ratas, esta modificación en el patrón de sueño es una consecuencia que podría estar vinculada en la generación de otros fenómenos asociados al estrés, dependientes del sueño MOR. La acupuntura es una herramienta terapéutica no farmacológica empleada desde hace miles de años en el tratamiento de múltiples trastornos. En últimas fechas reportes señalan su eficacia en el tratamiento de trastornos y síntomas asociados al estrés, como diversos tipos de insomnio, trastornos del estado de ánimo o incluso trastornos respiratorios durante el dormir, entre otros. Por ello, la OMS promueve la investigación básica y clínica de los efectos de la acupuntura. Para el caso de los trastornos del sueño y del estrés, la acupuntura ha utilizado, entre otros, la aplicación del estímulo en el punto Zusanli (ST36). Objetivo: Evaluar la modificación del patrón de sueño alterado por estrés por inmovilización (EI) mediante la aplicación del punto acupuntural ST36. Método: A ratas adultas macho de la cepa Wistar, fueron implantadas con un dispositivo para efectuar el registro polisomnográfico y les fue localizado el punto ST36, siendo marcado con tinta indeleble previo a la inmovilización y tratamiento. Se les sometió a EI por dos horas, al inicio de la etapa oscura y 90 minutos después se les aplicó una o dos agujas estériles previa técnica aséptica, por 30 minutos bajo el siguiente esquema: El conjunto de animales fue dividido en cinco grupos: A. Control (Intacto) $n=4$; B. $E I n=6$; C. $E I+$ punto sham $n=4 ; D$. $\mathrm{EI}+$ punto sham + punto ST36 $\mathrm{n}=4$; $\mathrm{E}$. $\mathrm{EI}+$ punto ST36 $\mathrm{n}=4$. Posteriormente se realizó un registro PSG por las 8 horas siguientes. Resultados: A los animales a los que se les aplicó el punto ST36, presentó un incremento significativo en el tiempo total de sueño. Particularmente, la etapa que presentó el aumento fue el SNMOR. En cuanto al SMOR, no hubo diferencias significativas; sin embargo, se observó tendencia de incremento de sueño MOR por parte del grupo únicamente inmovilizado. Conclusiones: La acupuntura sobre un punto específico (ST36) modifica el patrón de sueño con respecto al grupo control y a lo esperado por parte del grupo bajo estrés por inmovilización. A fin de fortalecer las tendencias observadas, habremos de incrementar el número de sujetos estudiados.

\section{Terapia acupuntural en tratamiento del trastorno de movimiento periódico de las extremidades durante el dormir}

Santana-Miranda $\boldsymbol{R},{ }^{*}$ RojasZamorano JÁ, ${ }^{\ddagger}$ Esqueda-León $E^{\mathcal{S}}$

*Clínica de Trastornos del Sueño, UNAM; ‡Área de neurociencias, UAMI; ${ }^{\S}$ Clínica de Trastornos de Sueño.DCBS. UAMI.

Antecedentes: Técnicas terapéuticas milenarias como la práctica de la acupuntura son utilizadas hoy día en todo el mundo, mostrando que son una alternativa no farmacológica a diversos padecimientos. Los trastornos de sueño son un área recién abordada por la medicina alópata; sin embargo, el tratamiento a diversas quejas asociadas a trastornos del dormir, ha sido contemplado por parte de la Medicina Tradicional China desde hace siglos. Recientes investigaciones reportan el efecto benéfico de la acupuntura en el tratamiento de trastornos del sueño, entre ellos el insomnio secundario a trastornos del estado de ánimo y en trastornos respiratorios durante el dormir. Objetivo: Evaluar el efecto acupuntural sobre el trastorno de movimientos periódicos de las extremidades. Método: Participaron 10 adultos (30 y 65 años), insomnes crónicos con sintomatología de síndrome de piernas inquietas (criterios clínicos) y presencia de trastorno de movimiento periódico de las extremidades (criterios polisomnográficos). Se les aplicaron pruebas psicométricas estandarizadas (Índice de Calidad de Sueño de Pittsburg, Escala para la Ansiedad de Hamilton y Escala para la Depresión de Beck) y fueron registrados polisomnográficamente por 8 horas previo al inicio del tratamiento. Se 
les aplicó agujas estériles y desechables previa técnica aséptica en los mismos puntos acupunturales bajo un esquema sindromático a todos los pacientes por 30 minutos, en dos sesiones vespertinas por semana hasta completar 10 sesiones en total, durante la terapia acupuntural los pacientes llevaron el registro de un diario de sueño. Al finalizar se repitió la aplicación de escalas y del registro polisomnográfico. Resultados: Los resultados preliminares muestran una significativa reducción del índice de PLMS, disminución significativa de los microdespertares y no se presentaron cambios en la arquitectura de sueño. Los resultados de las escalas que evaluaron calidad de sueño, nivel de ansiedad o presencia de síntomas depresivos presentaron una disminución significativa. Conclusiones: Los presentes resultados son consistentes con reportes previos sobre los cambios positivos tanto en parámetros objetivos como subjetivos de la acupuntura sobre trastornos del sueño; pero este estudio evidencia su utilidad como opción terapéutica en el área de los trastornos de movimiento durante el dormir.

\section{Efecto de la epilepsia del lóbulo temporal sobre el sueño}

\section{Ayala-Guerrero F, Mexicano-Medina G, Castro-Domínguez D, Lazo LA, Valencia-Flores $K$}

Laboratorio de Neurociencias, Universidad Nacional Autónoma de México

Introducción: Se ha descrito que la epilepsia afecta de manera significativa al ciclo vigilia-sueño. De acuerdo a su distribución nictemeral se han identificado 3 tipos diferentes de epilepsia:
Diurna, nocturna y difusa. Existen reportes describiendo la alteración del sueño en los pacientes que sufren de epilepsia nocturna. Esta alteración del sueño produce una sensación de cansancio y somnolencia diurna excesiva que afecta la calidad de vida de los pacientes. Aunque existen datos que muestran que las crisis convulsivas inducen la desorganización del sueño, éstos son frecuentemente incompletos y a veces contradictorios, por tal motivo implementamos un modelo animal que reproduce las características de la epilepsia de lóbulo temporal con el propósito de analizar su efecto sobre el sueño. Hipótesis: La epilepsia fragmentará el sueño y reducirá su cantidad. Métodos: Los estudios se realizaron en ratas adultas de la variedad Wistar implantadas crónicamente para llevar a cabo registros poligráficos. Se obtuvo un registro control durante 10 horas continuas, seguido de un registro similar después de la administración de ácido kaínico $(10 \mathrm{mg} / \mathrm{kg})$ para inducir crisis generalizadas. Resultados: Las crisis inducidas con ácido kaínico producen una inhibición total del sueño, seguido por una recuperación progresiva. Conclusiones: Estos hallazgos sugieren que las crisis generalizadas inducen un estado de excitación cerebral que afecta los mecanismos que participan en la regulación del sueño.

\section{Relacion del trastorno de movimientos periódicos de las piernas en 100 casos con apnea obstructiva}

\section{Alanis-Guevara MI, Pella-Cruzado $V$, Vásquez-Reyes $F$}

Hospital Ángeles del Pedregal, Ciudad de México, Centro Médico Nacional de Occidente de Jalisco; *Hospital Ángeles del Pedregal, Ciudad de México, Clínica Especializada de Epilepsia y Sueño de Tlalpan

Introducción: El trastorno de movimientos periódicos de las piernas (PLMD) se caracteriza por movimientos involuntarios repetitivos en sueño superficial, asociados o no a apnea obstructiva (SAOS), que ocasionan múltiples microdespertares y desestructuracion del sueño, manejados con relativo éxito con medicamentos y CPAP. Existe una asociacion aún no estimada entre PLMD y SAOS, así como su nexo con enfermedades metabólicas y la edad de recrudecimiento. Hipótesis: La correlacion PLMD y SAOS empeora con la edad y el tiempo de evolución. Objetivo: Evaluar la relacion PLMD y SAOS desde el punto de vista clínico como terapéutico. Métodos: Cohorte retrospectiva de 100 adultos con SAOS y PLM evaluados por video polisomnografía. Resultados: $100 \%$ con SAOS y PLM. Edad media 53 años, 82\% varones, divididos en tres grupos: Jóvenes (25\%, 18-30 años), adultos (51\%, 31-64 años) y seniles (mayores 65 años). Tiempo medio del padecimiento 10 años; mayor retraso en ancianos. Factores asociados: hipertensión $57 \%$, sobrepeso y obesidad $90 \%$, Mallampati severo $75 \%$, perímetro abdominal engrosado $86 \%$. Conclusiones: En todos los grupos, la relacion PLM y SAOS fue directamente proporcional en severidad y mejoraron con CPAP. La diabetes fue sobresaliente en ancianos.

\footnotetext{
$\triangle$ Correspondencia:

Dr. Luis Torre-Bouscoulet, Subdirector de Investigación Clínica, Instituto Nacional de Enfermedades Respiratorias Ismael Cosío Villegas.

Calzada de Tlalpan Núm. 4502, Colonia

Sección XVI, México D.F., 14080.

Tel (52) (55) 54871700; ext. 5160

Correo electrónico: luistorreb@gmail.com
} 